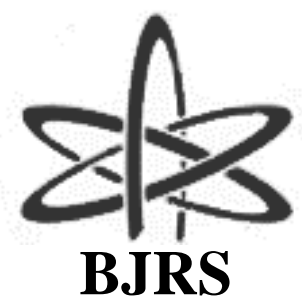

\author{
BRAZILIAN JOURNAL \\ $\mathrm{OF}$ \\ RADIATION SCIENCES \\ 07-2A (2019) 01-14
}

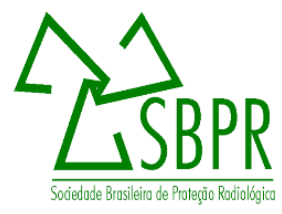

\title{
IMAGE QUALITY ANALYSIS OF AN INDUSTRIAL INSTANT NON-SCANNING TOMOGRAPHY SYSTEM WITH DIFFERENT COLLIMATORS
}

\author{
A. F. Velo ; A. G. Alvarez ${ }^{\text {a }}$ M. M. Hamada ${ }^{\text {a }}$ C. H. Mesquita ${ }^{\mathrm{a}}$ \\ ${ }^{a}$ Instituto de Pesquisas Energéticas e Nucleares/ Centro de Tecnologia das Radiações. 05508-000, São Paulo, São \\ Paulo, Brasil \\ afvelo@usp.br
}

\begin{abstract}
An instant non-scanning tomography system is being developed at the IPEN. This type of tomography system, due to its configurations, provides a low spatial resolution; however, it presents a high temporal resolution as an advantage. In this sense, it has high applicability in industrial processes involving dynamic multiphase processes, like distillation columns. Thereby, to obtain the best conditions of image acquisition, this tomography system comprising different collimators was simulated with Monte Carlo using the MCNP4C.The image quality was evaluated with Matlab® 2013b analyzing the contrast to noise ratio (CNR), root mean square ratio (RMSE), signal to noise ratio (SNR) and the spatial resolution by the Modulation Transfer Function $(\operatorname{MTF}(f))$, to identify which collimator fits better to the tomography in development. It was simulated three situations; (i) with no collimator; (ii) $\varnothing 25 \mathrm{x} 50 \mathrm{~mm}^{2}$ cylindrical collimator with a septum of $\varnothing 5.0 \times 50 \mathrm{~mm}^{2}$; (iii) $\varnothing 25 \times 50 \mathrm{~mm}^{2}$ cylindrical collimator with a slit septum of $24 \times 5.0 \times 50 \mathrm{~mm}^{3}$. RMSE values for no collimator presented better results. CNR showed that no collimator and slit collimator reaches the same CNR values, but no collimator decreases more than the slit collimator as the number of iteration rises. The hole collimator reaches a higher CNR value, however decreases more than the others. The spatial resolution with no collimator and slit collimator were around $31.9 \mathrm{~mm}$, and for the hole collimator was around $20 \mathrm{~mm}$
\end{abstract} Keywords: Collimators, Industrial Tomography, Monte Carlo Simulation.

ISSN 2319-0612

Accepted: 2018-12-11 


\section{INTRODUCTION}

The greatest impact of the computed tomography (CT) applications currently occurs in medicine. In industry there is much interest of using CT in order to know the interior of: (i) industrial objects; (ii) machines and their means of production. The purpose of this tomography is to: (a) control the quality of the final product and (b) optimize production and analyze the quality of the means of production.

Some industrial processes, like distillation columns, involve fast dynamic processes containing solid, liquid and gas mixtures. These distillation columns are usually built with steel and have large diameters and thicknesses that make their analysis unfeasible with conventional X-ray beams [1-3]. For this reason, gamma radioactive sources in the energy ranges of $317 \mathrm{keV}\left({ }^{192} \mathrm{Ir}\right), 662 \mathrm{keV}\left({ }^{137} \mathrm{Cs}\right)$ to $1250 / 1332.501 \mathrm{keV}\left({ }^{60} \mathrm{Co}\right)$ are preferable instead X-rays [3].

In addition, scan systems, like computed tomography (CT), should be adapted for different size of objects that are usually sited in a hostile environment, containing flammable superheated materials, occasionally subjected to high internal pressure and presenting many difficulties for placing devices around these objects. Besides, the phenomena related to multiphase processes are usually fast, requiring high time resolution of the CT data acquisition devises [1-4]. In such case, ideally, the CT system should be fixed and not need to move their sources and detectors around the object, for this, Instant Non-Scanning and fifth generation tomography meet these requirements [5, 6]. Additionally, the system should be light enough to be portable and easily installed.

In order to be applicable in industrial plants, an Instant Non-Scanning tomography system is being developed at IPEN (Instituto de Pesquisas Energéticas e Nucleares). This system comprises 5 sets of fourteen 1x2 inches (diameter and length) $\mathrm{NaI}(\mathrm{Tl})$ detectors and five shielding cases for radioactive sources. Each shielding case is placed diametrically opposite to a fan detector set as showed in Fig. 1 [3].

The $\mathrm{NaI}(\mathrm{Tl})$ detectors are capable to detect large range of energies, i.e. from $59.5412 \mathrm{keV}{ }^{241} \mathrm{Am}$ to $1250 / 1332.501 \mathrm{keV}{ }^{60} \mathrm{Co}$ [7], then the choose of the source to be used depends on the material densities, wall thickness and dimension of the object to be evaluated by tomography measurements. Also, the proposed tomography system can be adjusted to the column or pipes by changing the 
number of detector set. Thus, the tomography system has the capacity of being adapted and applied for different object types, such as, column or pipe sizes found usually in the industrial plants. The tomography system can be mounted on a wooden platform, which is lightweight to be replaced in future applications according to the challenges of new geometry, dimension of the objects and application requirements [3].

Figure 1: Instant Non-Scanning tomography design for multiphase analysis. NaI(Tl) detectors (A), radioactive shielding case $(B)$, wooden platform $(C)$, the multiphase object to be analyzed $(D)$ and detector collimator $(E)[3]$.

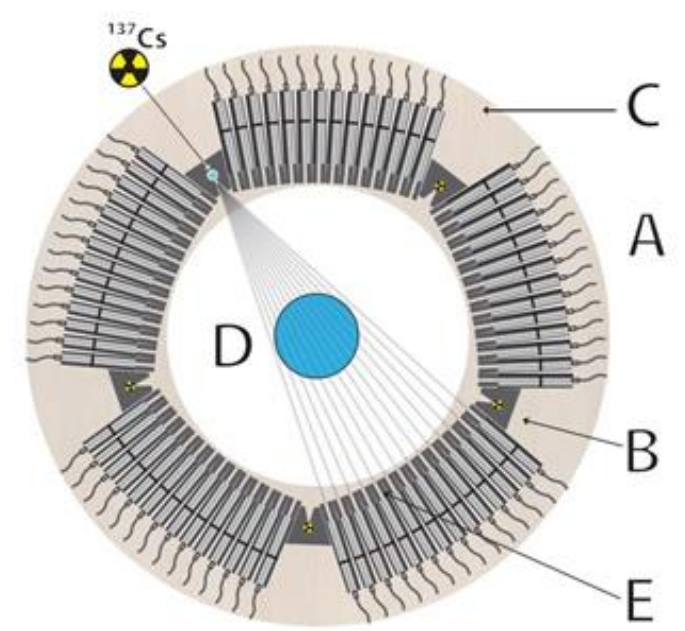

The Instant Non-Scanning Tomography system, due to its configurations, provides a low spatial resolution; however, it presents a high time resolution as an advantage [3]. In this sense, it has high applicability in industrial processes involving dynamic multiphase processes, like distillation columns [3]. Because it presents low spatial resolution, it is important to study the best conditions of data acquisition to improve its performance. In this way some studies using simulation is vital before finishes the tomography system.

The Monte Carlo N-Particle code (MCNP) is a Monte Carlo radiation transport, developed by Los Alamos National Laboratory [8], and designed to track different type of particles (neutrons, electrons, gamma rays, etc.) over a range of energies [9]. The code simulates individual particle trajectories, since its emission up to the energy cut-off, and record aspects of their average behavior $[3,9]$. The particle energy is transferred to the matter by absorption, escape, physical cut-off, and others [9]. Probability distributions are randomly sampled using transport data to determine the outcome at each step of its trajectory [8-10]. The MCNP code can be used to simulate gamma-rays interactions which comprise: i) incoherent and coherent scattering; ii) the possibility of fluorescent 
emission after photoelectric absorption; iii) pair production with local emission of annihilation radiation and Bremsstrahlung effect $[3,9,10]$.

In the present work, the MCNP was used to simulate the Instant Non-Scanning Tomography system with different types of collimators to analyze the effect of these collimators on the quality of the images obtained by the tomography system simulated, in order to study with collimator improves the performance of the system that is being developed.

\section{MATERIALS AND METHODS}

The simulation was carried out with five sets of $\mathrm{NaI}(\mathrm{Tl})$ detectors ( 1 x 2 inches) and five $662 \mathrm{keV}$ ${ }^{137} \mathrm{Cs}$ sources enclosed in a tungsten shielding cases. For this purpose, each set is constituted of fourteen $\mathrm{NaI}(\mathrm{Tl})$ detectors, that is positioned diametrically opposite to a source shielding case as shown by Fig. 1. All five detector sets and the five radioactive source shielding cases are mounted on a wooden platform as shown in Fig. 1.

Three situations were performed; (i) with no collimator; (ii) $27 \mathrm{~mm}$ x $50 \mathrm{~mm}$ collimator with a septum of $\varnothing 5.0 \mathrm{~mm} \times 50 \mathrm{~mm}$ and (iii) $27 \mathrm{~mm} \times 50 \mathrm{~mm}$ collimator with a slit septum of $24 \mathrm{~mm} \times 5.0$ $\mathrm{mm} \times 50 \mathrm{~mm}$. The schemes of the collimators are presented by Fig 2.

Figure 2: Proposed simulation (a) no collimator; (b) slit collimator and (c) hole collimator
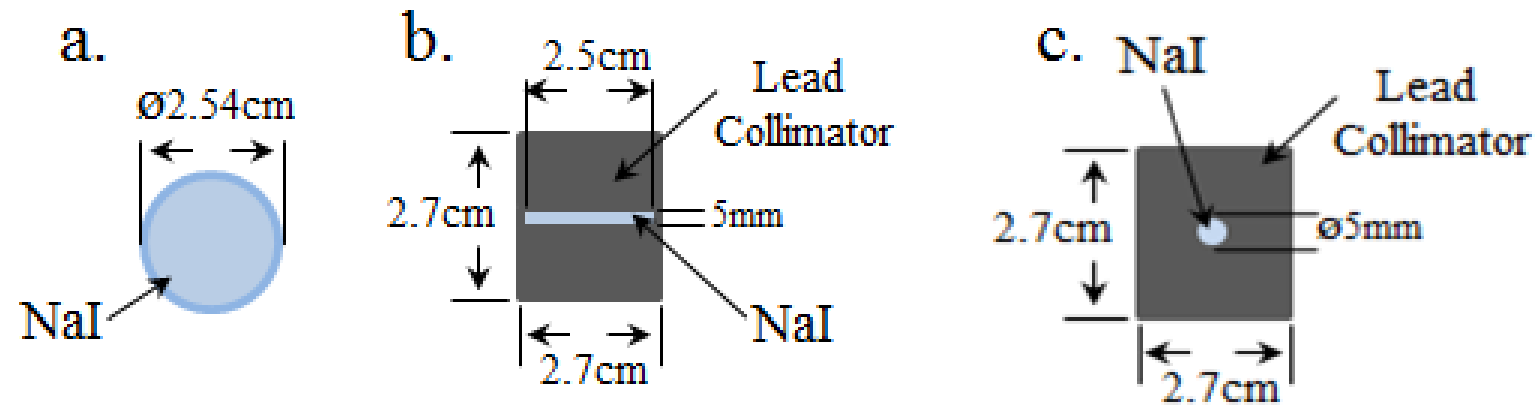

When performing the simulation of $\mathrm{NaI}(\mathrm{Tl})$ detectors, some corrections should be made to improve the simulation approaching to the real case. Two of the main corrections are essential: (i) the 
determination of the photon detection efficiency; and (ii) the energy resolution, which is related to distinguish different peaks very close to each other in the energy spectrum $[9,11]$.

The energy resolution of the detector is given by the full width at half maximum (FWHM), which is approximated to a Gaussian peak for a given energy $[9,10]$. Some of the effects related to the photopeak are inherent to the electronic circuit of the spectrometric system which is not simulated by the MCNP. In this way, to optimize the detector response and consider these physical effects in the simulation, it is necessary to obtain experimentally adjustment parameters of the detector energy resolution and apply a MCNP code function to fits the Gaussian to the spectrum obtaining the proper corrections [9].

The MCNP fitting technique to take into account the resolution of the real detector, measured experimentally, consists of using a "FT8 GEB" card into the input file of the code [3, 9]. GEB (Gaussian energy broadening) is a special treatment for tallies to better simulate a physical radiation detector $[3,9]$.

A non-linear function adjusted by least-squares procedure was used as an input to the MCNP code, using a fitting function [10]. These parameters should be used with the (GEB) command in order to consider the energy resolution of the detector in the simulation $[9,10]$. These parameters were calculated in previous work [3].

The detector simulation was based on the dimensions of the real $\mathrm{NaI}(\mathrm{Tl})$ detector used in the Instant non-scanning tomography.

For the estimation of the pulse height, a F8 tally was applied on MCNP code to obtain the deposited energy distribution per incident photon on the $\mathrm{NaI}(\mathrm{Tl})$ detector, where for each individual history, the tally accumulates the deposited energy. In order to obtain a good statistic counts, the histories number used was $11 \mathrm{E}+09$.

A phantom was simulated to evaluate the physical parameters of the Instant Non-canning tomography system [3]. The phantom was composed of a cylindrical polymethylmethacrylate (PMMA $((\rho \approx 1.19 \mathrm{~g} / \mathrm{cm} 3))$ of $\varnothing 24 \mathrm{~cm} \times 20 \mathrm{~cm}$ dimensions and an aluminum cylindrical bar $(\rho \approx$ $2.698 \mathrm{~g} / \mathrm{cm} 3)$ of $\varnothing 7.5 \mathrm{~cm} \times 20 \mathrm{~cm}$ inside the PMMA. The geometry is illustrated by Fig. 3. 
Figure 3: Simulated phantom for physical analysis

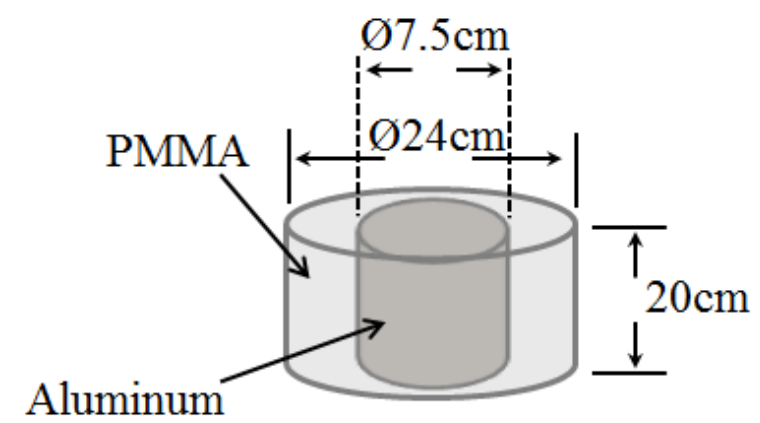

The Root Mean Square Error (RMSE) was measured to evaluate which algorithm of reconstruction approaches the pixel values that correspond to the linear attenuation coefficient, obtained by the reconstruction, to the theoretical values. This method is widely used to measure the quality of the image. The RMSE was calculated by the equation (1) [3].

$$
R M S E=\sqrt{\frac{\sum_{i=1}^{N}\left(\mu_{i}-\widehat{\mu}_{l}\right)^{2}}{N}}
$$

where $\mu_{i}$ is the experimental pixel value obtained, $\widehat{\mu_{l}}$ is the theoretical linear attenuation coefficient. The theoretical image is presented by the Fig. 4.

Figure 4: Theoretical image

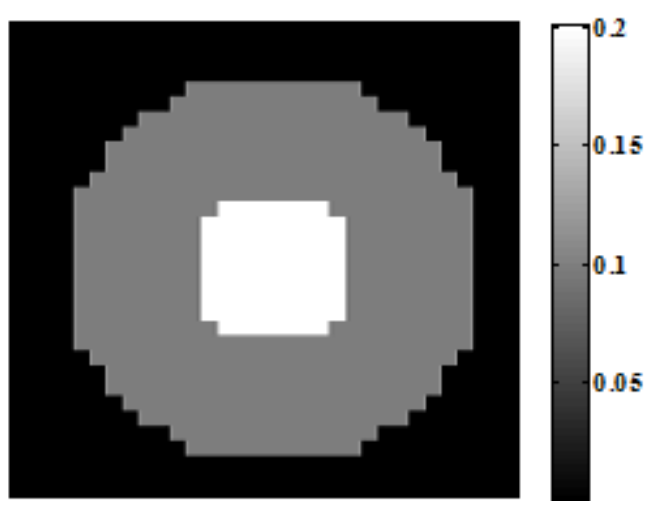

The Contrast to Noise Ratio (CNR) was measured placing regions of interest (ROIs) in the images obtained by the phantom (Fig. 2) with the different algorithms. A circular ROI was placed over the 
cylinder corresponding the aluminum (ROI A) and another ROI, with the same size of the ROI A, placed over the background, corresponding the PMMA (ROI B), then the values of the CNR was obtained by the equation (2) [12].

$$
C N R=\frac{\left|\overline{\mu_{A}}-\overline{\mu_{B}}\right|}{\sqrt{\frac{\sigma_{A}^{2}+\sigma_{B}^{2}}{2}}}
$$

where $\overline{\mu_{A}}$ is the mean pixel value of the ROI A, $\overline{\mu_{B}}$ is the mean pixel value of the ROI $\mathrm{B}, \sigma_{A}$ is the standard deviation of ROI A and $\sigma_{B}$ is the standard deviation of the ROI B. The ROIs is represented on Fig. 5, where the red circle represents the ROI A and the blue circle represents the ROI B.

Figure 5: ROIs used to measure the CNR
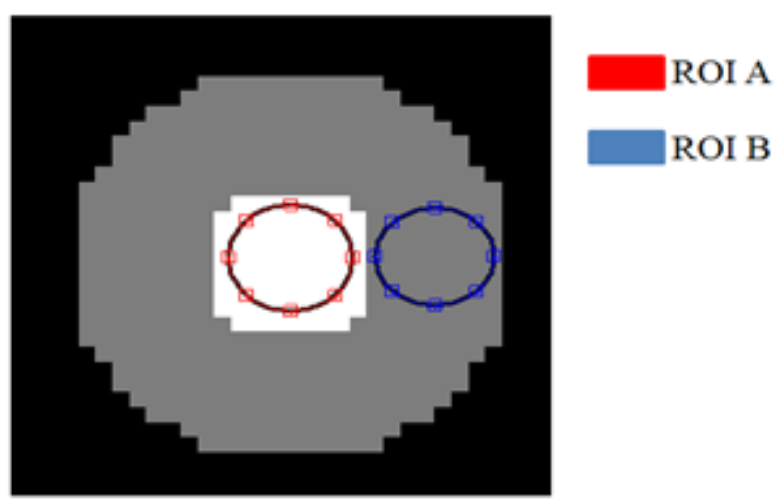

The most comprehensive metric used to measure and report spatial resolution of imaging systems is the modulation transfer function (MTF) [12-14]. Conventionally, the spatial resolution is estimated as the inverse of the value at $10 \%$ of MTF curve [12-14]. In the present work, MTF was calculated using the Edge Spread Function, commonly known as ESF parameter [14, 15].

The algorithm used to reconstruct the images was the Maximum Likelihood Expectation Maximization (MLEM) [16], in a matrix grid of 32x32. All physical measurements and reconstructions were performed by Matlab 2013b®. 


\section{RESULTS AND DISCUSSION}

The ${ }^{137} \mathrm{Cs}$ spectrum obtained by the simulated $\mathrm{NaI}(\mathrm{Tl})$ detectors with no collimator, slit collimator and hole collimator are shown by Fig. 6.

Figure 6: Simulated ${ }^{137}$ Cs spectrum for no collimator, slit collimator and hole collimator

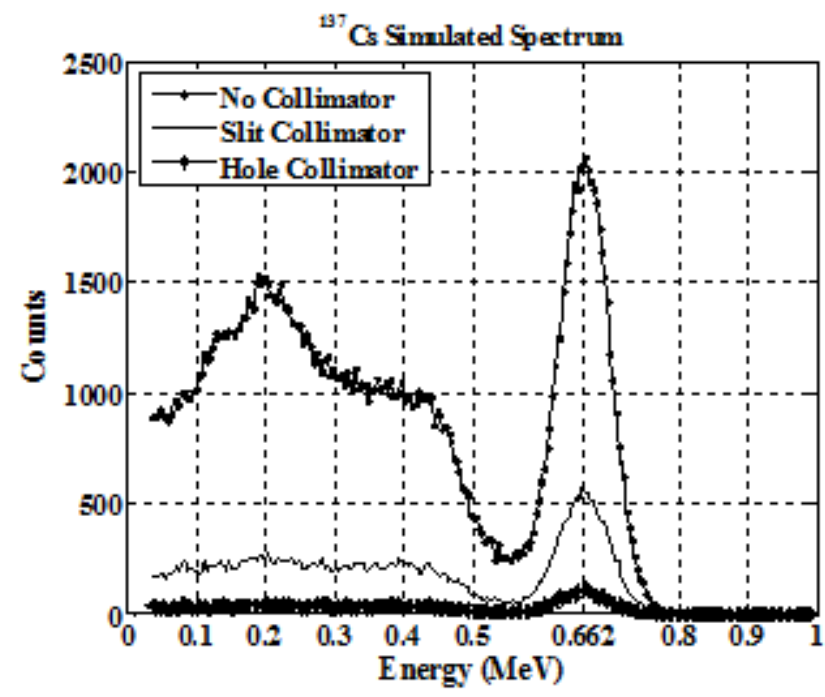

By Fig. 6 it is possible to observe that for the simulation with no collimator the sensitivity is higher than with collimators, and the ${ }^{137} \mathrm{Cs}$ spectrum obtained by the simulation with slit collimator has higher sensitivity then the spectrum obtained by the hole collimator. The reconstructed images of the phantom (Fig. 3) obtained by the simulation of the Instant Non-Scanning tomography with different collimators are presented by the Fig. 7, where Fig. 7a. is the simulation with no collimator; Fig. 7b. is the simulation with slit collimator and Fig. 7c. is simulation with hole collimator.

The RMSE parameter applied to perform the quality of the image for the three different simulations was calculated using equation (1), comparing the experimental images with the theoretical image. Fig. 8 shows the curve behavior for each simulation. 
Figure 7: Reconstructed images of the simulated instant non-scanning with, (a) no collimator; (b) slit collimator and (c) hole collimator.

a.

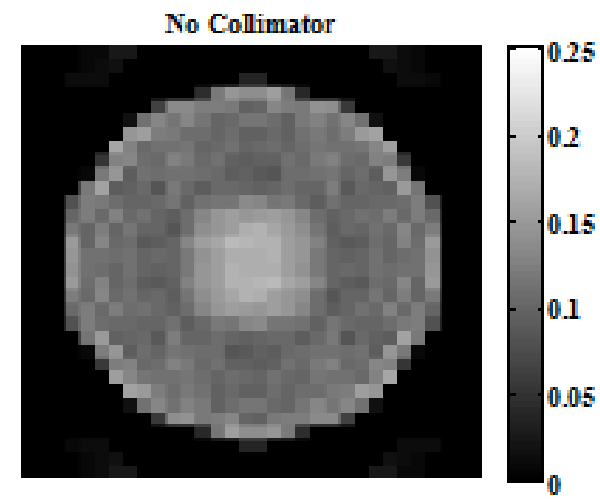

b.

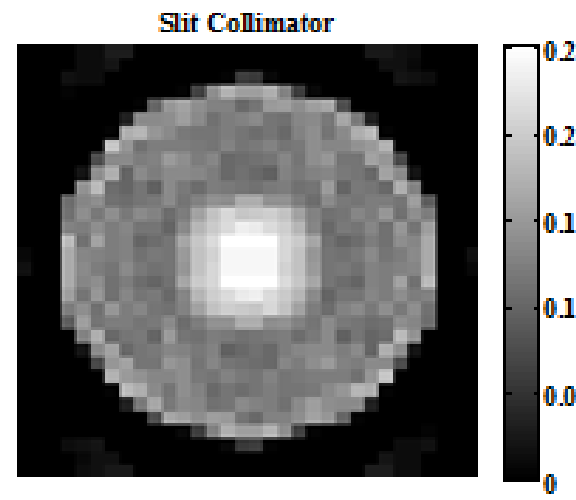

c.

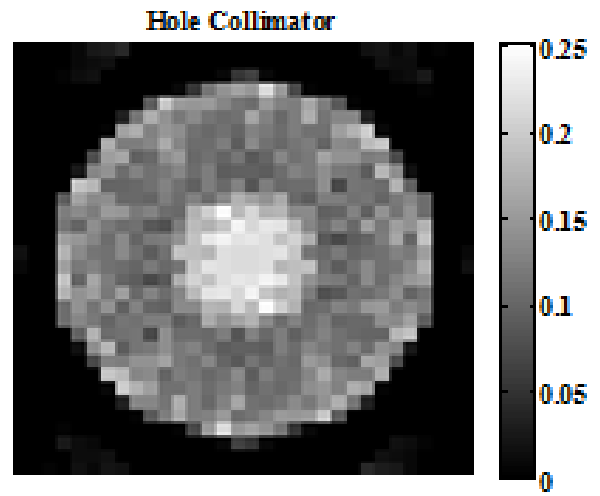

Figure 8: RMSE analysis for (a) no collimator; (b) slit collimator and (c) hole collimator

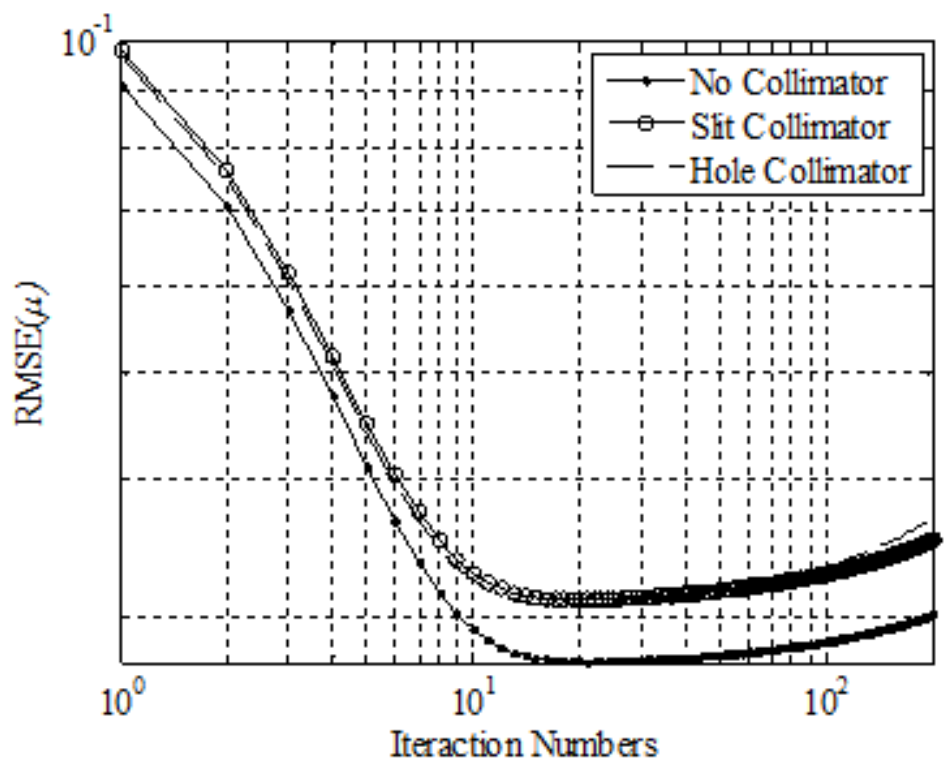


Fig 8 shows that the simulation with no collimator approaches to the theoretical value more than the simulation with collimators, and the data obtained by the simulation with collimators did not presented any differences on the RMSE value as the number of the iterations rises.

The analysis of the noise influence on the quality of the image was performed by the CNR parameter on the three simulations proposed. The results are shown by the Fig. 9. By this Fig., it is possible to observe that even the data obtained by the simulation with no collimator and with the slit collimator reaches the same CNR value in the first iterations, this values decreases rising the number of the iterations, however the CNR of the simulation with no collimator decreases more than the slit collimator due to the growth of the noise. The CNR for the hole collimator reaches a higher CNR value, however as the number of iterations rises, the noise decreases this value.

Figure 9: CNR analysis for (a) no collimator; (b) slit collimator and (c) hole collimator

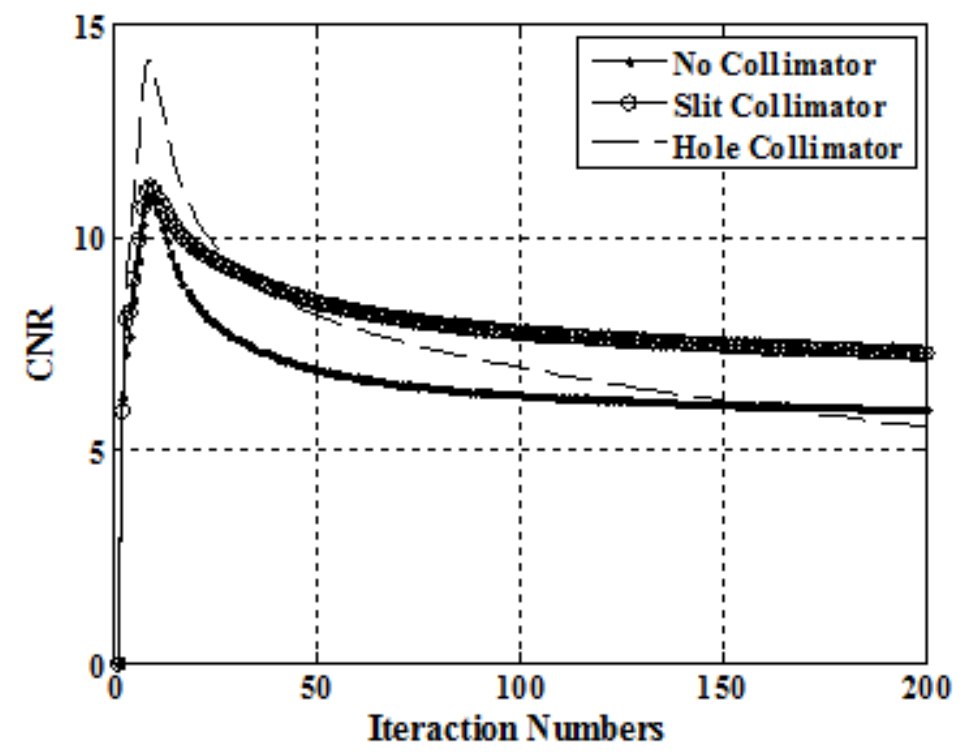

The spatial resolution of the reconstructed images was measured by the MTF (f). The MTF curves by the frequency spectrum (plmm-1) of the simulations proposed of the iteration number of 200 are presented by the Fig. 10. 
Figure 10: MTF analysis for (a) no collimator; (b) slit collimator and (c) hole collimator

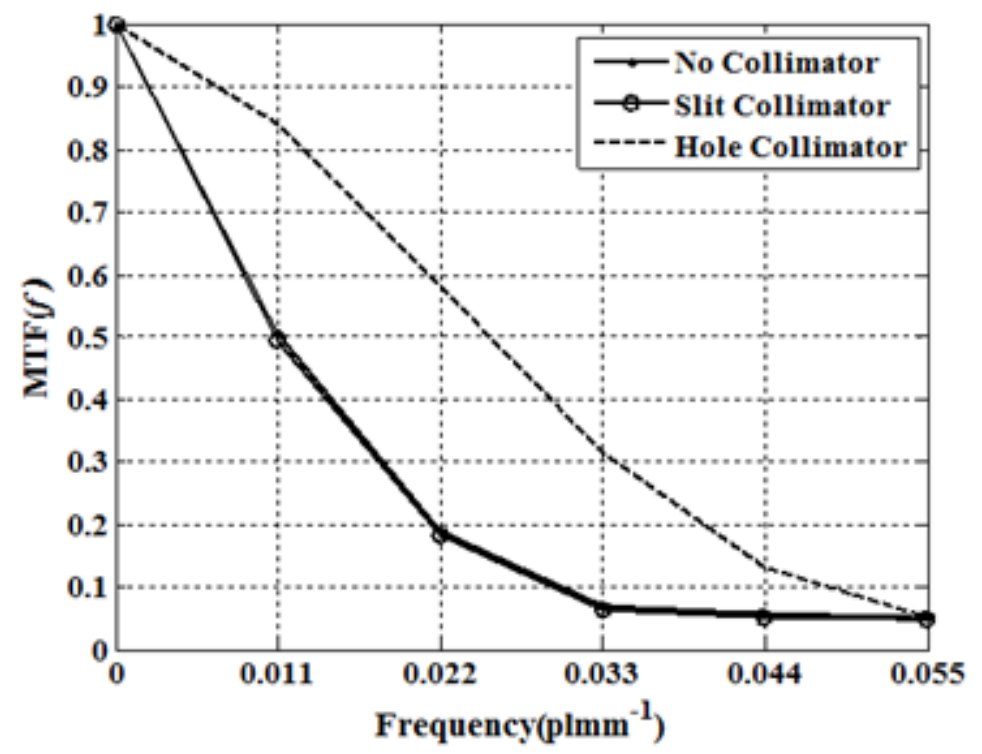

By the Fig. 10, the data obtained by the hole collimator simulation presents a better spatial resolution in all frequency spectrum compared to the others simulations proposed, which presented the same MTF value for all the frequency range. The maximum spatial resolution of the system is measured at $10 \%$ of the MTF, in other words, the spatial resolution is calculated by the inverse of the frequency in $10 \%$ of the MTF. Thereby the resolution for the simulation of no collimator, slit collimator is about of $31.9 \mathrm{~mm}$ and for the hole collimator is about $20 \mathrm{~mm}$.

\section{CONCLUSION}

The results of the RMSE values showed that the data obtained with no collimator approaches to the theoretical values more compared to the other two simulations, which means that the experimental linear attenuation coefficient is close to the theoretical values.

The CNR analysis showed that for the data acquired with no collimator and with the slit collimator reaches the same CNR value, but the data with no collimator decreases more than the slit collimator as the number of iteration rises, which means that no collimator suffer more noise growth than the data with the slit collimator. The data acquired with the hole collimator reaches a higher CNR 
value, however decreases mores than the other simulations, evidencing that the noise corrupt more the image with this kind of collimator compared to the others.

The spatial resolution of the instant non-scanning tomography system obtained with no collimator and with the slit collimator was the same for both, around $31.9 \mathrm{~mm}$, and for the hole collimator was around $20 \mathrm{~mm}$, which means that this last simulation proposed presents a better spatial resolution.

\section{ACKNOWLEDGMENT}

The authors express their acknowledgment to CNEN, FAPESP and IAEA for the financial support. The authors, Carlos Henrique de Mesquita and Margarida Mizue Hamada thank to CNPq and Alexandre França Velo to CNEN for their fellowship.

\section{REFERENCES}

[1] CHAOUKI, J, LARACHI, F, DUDUKOVIC, M.P. Non-Invasive Monitoring of Multiphase Flows. In: CHAOUKI, J, LARACHI, F, DUDUKOVIC, M.P Computerassisted gamma and X-ray tomography: Application to multiphase flow In NonInvasive Monitoring of Multiphase Flows, $1^{\text {st }}$ ed.; Elsevier: Amsterdam, The Netherlands, 1997; p 47 - 97.

[2] ISMAILA, I., GAMIOB J.C. Tomography for multi-phase flow measurement in the oil industry. Flow measurement and instrumentation. v. 16, p. 145-155, 2002.

[3] VELO A.F, HAMADA M.M., CARVALHO D.V.S., MARTINS J.F.T, MESQUITA C.H., A portable tomography system with seventy detectors and five gamma-ray sources in fan beam geometry simulated by Monte Carlo method. Flow measurement and instrumentation. v. 53, p. 89-94, 2017.

[4] JOHANSEN, G. A.; JACKSON, P. Radioisotope Gauges for Industrial Process Measurements. John Wiley \& Sons, 2004. 
[5] MAAD, R., JOHANSEN G.A. Experimental analysis of high-speed gamma-ray tomography performance. Measurement science and Technology, v. 19, p. 1-10, 2008,

[6] FISCHER F, HOPPE D, SCHLEICHER E, MATTAUSCH G, FLASKE H, BARTEL R AND HAMPEL U. An ultra-fast electron beam x-ray tomography scanner. Measurement science and technology, v. 19, p. 1-11, 2008.

[7] Disponível em <https://www.inl.gov/>. Acesso em 06 jan 2018.

[8] PELOWITZ, D.B. MCNP-X TM User's Manual, Version 2.5.0. 2005. LA-CP-05-0369. Los Alamos National Laboratory.

[9] SALGADO, C.M., BRANDÃO L.E.B., SCHIRRU, R., PEREIRA, C.M.N.A., CONTI C.C. Validation of a NaI(Tl) detector's model developed with MCNP-X code. Progress in Nuclear Energy, v. 59, p. 19-25, 2012.

[10] HADIZADEH H., MOWLAVI, A.A., THOMPSON, M.N., HAKIMABAD, H. M. Proper shielding for $\mathrm{Na}(\mathrm{Tl})$ detectors in combined neutron-gamma fields using MCNP. Nuclear Instruments and Methods in Physics, v. 522, p. 447-454, 2004.

[11] JEHOUANI, A., ICHAOUI, R., BOULKNEIR, M. Study of the NaI(Tl) efficiency by Monte-Carlo method. Applied Radiation and Isotopes, v. 53, p. 887-89, 2000.

[12] CHRISTIANSON O., CHEN J. J.S., SAIPRASAD G., FILliBEN J.J, Peskin A., TRIMBLE C., SEIGEL E L., SAMEI E. An improved index of image quality for taskbased performance of CT iterative reconstruction across three commercial implementations. Radiology, v. 275, p. 725-734, 2015

[13] SMITH, STEVEN W. The scientist and engineer's guide to digital signal processing. $2^{\text {nd }}$ ed, San Diego, California Technical Publishing, 1999.

[14] RICHARD S.L, HUSARIK D.B., YADAVA G., MURPHY S.N., SAMEI E. Towards task-based assessment of CT performance: System and object MTF across different reconstruction algorithms. Medical Physics, v. 39, p. 4115 - 4122, 2012. 
[15] SAMEI E., FLYNN M.J., REIMANN D.A. A method for measuring the pre- sampled MTF of digital radiographic systems using an edge test device. Medical. Physics, v. 25, p. 102-113, 1998.

[16] MAAD R., HJERTAKER B.T., JOHANSEN G.A. and OLSEN Ø. Dynamic characterization of a high speed gamma-ray tomography. Flow Measurement and Instrumentation, v. 21, p. 538-545, 2010. 